\title{
Cost-Effectiveness of Early Oral Feeding Following Total Gastrectomy
}

\author{
Akinori Hisashige $^{1}$, Nobuyuki Shimizu ${ }^{2} \&$ Yasuyuki Seto $^{3}$ \\ ${ }^{1}$ The Institute of Healthcare Technology Assessment, Tokushima, Japan \\ ${ }^{2}$ Department of Surgery, International University of Health and Welfare, Sanno Hospital, Tokyo, Japan \\ ${ }^{3}$ Department of Gastroenterological Surgery, Graduate School of Medicine, the University of Tokyo, Tokyo, \\ Japan \\ Correspondence: Akinori Hisashige, Institute of Healthcare Technology Assessment, 2-24-10, Shomachi, \\ Tokushima, 770-0044, Japan. Tel: 81-88-631-3313
}

Received: September 24, 2020 Accepted: November 19, 2020 Online Published: November 26, 2020

doi:10.5539/gjhs.v13n1p64

URL: https://doi.org/10.5539/gjhs.v13n1p64

\begin{abstract}
Background: Gastric cancer is a major health problem worldwide. Effectiveness of early oral feeding (EOF) following gastrectomy has been internationally evaluated, even though there is limited evidence available. In Japan, recently, a randomized controlled trial of EOF following total gastrectomy showed a decreased length of hospital stay, compared with conventional oral feeding (COF). To evaluate value for money of EOF following gastrectomy, cost-effective analysis was carried out based on this trial.
\end{abstract}

Methods: The analysis was carried out from a societal perspective as the base case. The subjects were randomly assigned to the EOF group $(\mathrm{N}=32)$ and the COF group $(\mathrm{N}=30)$. While the EOF group received a special diet (i.e., iEAT) from the postoperative day (POD) 1 to POD 3, the COF group received conventional diet (i.e., a liquid diet or rice gruel) on POD 4 and thereafter. The observation period was 3 weeks after total gastrectomy. As an effectiveness measure, quality-adjusted life days (QALDs) were estimated. Quality of life for health conditions was evaluated by using EuroQol-5Dimentions (EQ-5D)-3Levels among the subjects. Costs (e.g., nutritional support, hospitalization and treatment of adverse events) were estimated from trial data during observation. Qualitative and stochastic sensitivity analyses were performed to examine the robustness of the results.

Results: The mean QALDs per patient for the EOF and the COF groups were 14.93 and 14.19, respectively. Incremental effectiveness of the EOF group to the COF group was 0.74 QALDs ( $\mathrm{p}=0.359$, t-test). The mean total cost per patient in the EOF and the COF groups was $\$ 3,177.7$ and $\$ 3,755.4$, respectively. Incremental cost was $-\$ 577.7$ ( $\mathrm{p}=0.03$, t-test, Welch). This means EOF is dominant (cost saving). The qualitative sensitivity analysis and the stochastic sensitivity analysis showed the relative robustness of these results.

Conclusions: Economic evaluation of EOF following total gastrectomy showed that EOF was cost-saving, with non-significant increase in effectives (i.e., QALD), compared with COF. This result can contribute to strengthen the evidence for wide use of EOF.

Keywords: early oral feeding; gastric cancer; gastrectomy; cost-effectiveness; quality-adjusted life days; EQ-5D

\section{Background}

Gastric cancer is a major health problem worldwide. It ranks third in all causes of death from cancer, with about 9.3 million deaths in 2018 (Bray et al., 2018; Rawla \& Barsouk, 2019). In Japan, although its mortality ranked also third in 2018 and has decreased in recent years, it still has the highest incidence despite advances in prevention (The Editorial Board of the Cancer Statistics in Japan, 2018). As to treatment strategies, while complete resection is the only unimodal treatment with curative intent, surgery is a common treatment for all stages of gastric cancer (Coburn et al., 2018).

Recently, enhanced recovery after surgery (ERAS), or fast-track surgery, has been proposed and increasingly attracted attention (Gramlich et al., 2016; Paton et al., 2014). ERAS is a comprehensive and multidisciplinary framework for accelerating recovery during perioperative care and has been implemented mainly for colorectal surgery and spread to other areas including gastrectomy (Mortensen et al., 2014). An evidence synthesis of 17 systematic reviews and 12 randomized controlled trials (RCTs) showed that ERAS can reduce the length of hospital stay by $0.5-3.5$ days without increasing readmission rate (Paton et al., 2014). In gastric cancer surgery, 
also, similar results to those in ERAS were observed (Bermish, Chan, Blake, Karran \& Lewis, 2015; Li, Wang, Li, Bai, \& Zhao, 2017).

Although it has not been identified what component mainly contributes to the effectiveness of ERAS, early oral feeding (EOF) is considered as one of the most important elements of ERAS (Mortensen et al., 2014). While EOF usually initiates oral feeding of water or liquid diet on the post operative day (POD) 1 before flatus and shifts to ordinary diets, conventional oral feeing (COF) usually initiate this oral feeding on POD3 or after flatus and gradually shifts to ordinary diets (Liu et al., 2014; Tweed et al., 2019). EOF for gastric cancer surgery has been widely diffused and implemented, and recently systematic reviews of EOF following gastrectomy, based on RCTs, were reported (Liu et al., 2014; Tweed et al., 2019). These reviews showed that EOF after gastrectomy shortened the length of hospital stay without increasing postoperative adverse events (AEs) or readmissions.

Recently, a RCT in Japan has been conducted to evaluate the efficacy and safety of EOF following total gastrectomy for gastric cancers, and showed that EOF shortened the length of hospital stay without increasing adverse events (Shimizu et al., 2018). Since there has not been any full economic evaluation of EOF following gastrectomy, based on a RCT, we conducted a study to evaluate the cost-effectiveness of this intervention.

\section{Methods}

\subsection{Analytical Overview}

Cost-effective analysis was conducted based on the RCT evaluating the effect of EOF following total gastrectomy among gastric cancer patients (Shimizu et al., 2018). The information on its methods and results has been presented in detail elsewhere (Shimizu et al., 2018).

Patients who underwent total gastrectomy were randomly assigned to either an intervention group (early oral feeding, EOF) $(\mathrm{N}=32)$ or a control group (conventional oral feeding, $\mathrm{COF})(\mathrm{N}=30)$. The EOF group was given iEAT ${ }^{\circledR}$ from lunch on POD 1 through dinner on POD 3. On POD 4 and thereafter, ordinary hospital diets were provided. iEAT ${ }^{\circledR}$ (EN Otsuka Pharmaceutical Co., Ltd., Japan) is a commercially available food, and its physical properties have been adjusted for shape-retention despite its softness. Although it retains an appearance similar to ordinary food, it can be disintegrated by the tongue. In a prior study that dissolved different foods in artificial digestive juices into small residues, iEAT was shown to disintegrate more quickly than ordinary foods (Higashiguchi, 2013).

In this study, iEAT offered as EOF consisted of one staple food (rice) and two side dishes. Its average calories and protein amount were approximately $450 \mathrm{kcal}$ and $25 \mathrm{~g}$ per meal, respectively.

As to an intervention, iEAT was used, instead of diets in conventional EOF, to enhance the effect of EOF. Its reasons are as follows. Firstly, it offers standardized intervention, since diets in conventional EOF vary from liquids to rice porridge. Secondly, the amount of nutrition intake of energy and protein for iEAT were significantly higher than those for conventional diets mentioned before (Higashiguchi, 2013). It is pointed out that patients after gastroenterological surgery require high energy and protein to prevent complications and accelerate recovery (Fukatsu, 2019).

On the other hand, the COF group received conventional nutritional management. The COF was usually started with a liquid diet or rice gruel on POD 3 or 4 and shifted to ordinary diets on POD 7 or 8 , although it varied depending on the facility. Liquid diet or rice gruel in COF typically consisted of three dishes in both meals. Their average calories and protein amount were approximately $260 \mathrm{kcal}$ and $8.3 \mathrm{~g}$ per meal, and $430 \mathrm{kcal}$ and $18 \mathrm{~g}$ per meal, respectively. For both groups, there were no limitations regarding infusions, eating between meals, and other means of perioperative management.

As is shown in Table 1, between the EOF group and the COF group, no statistical differences were observed in age, sex, height, clinical state, kind of surgery and operating time. As to clinical outcomes, a statistically significant difference was observed in feeding start day $(\mathrm{p}<0.01)$, and days reaching the specified oral energy intake $(\mathrm{p}<0.05)$. The length of hospital stay in the EOF was significant shorter than that in the COF $(p<0.05)$, while there was no statistical difference in the proportion of adverse event between two groups. In this paper, all data were presented based on per-protocol instead of intention-to-treat, according to the research protocol predefined.

As a type of economic analysis (Drummond, Sculpher, Claxton, Stoddart, \& Torrance, 2015), a cost-effective analysis was performed. Costs and effectiveness were evaluated and compared between the EOF and the COF groups. A societal perspective was adopted as a perspective of economic analysis to evaluate value for money of EOF compared to COF (Drummond et al., 2015). The effectiveness measure used was quality-adjusted life-days (QALDs) (Ioannidis \& Graber, 2011). As to cost items, direct medical care costs (e.g., costs of nutrition, 
hospitalization and treatment of adverse events) were examined. Indirect costs (i.e., time costs or production loss among patients) after discharge were examined for a base case analysis. However, they were excluded in the sensitivity analysis mentioned later. As a time horizon for evaluation, 3-week follow-up after gastrectomy was considered.

Table 1 Characteristics of subjects and interventional outcomes

\begin{tabular}{|c|c|c|c|}
\hline Item & $\operatorname{EOF}(N=32)$ & $\operatorname{COF}(\mathrm{N}=30)$ & p value \\
\hline Sex (male) & $78.1 \%$ & $73.3 \%$ & $0.566^{\mathrm{a}}$ \\
\hline Age (years) & $67.6(7.3)$ & $66.3(9.8)$ & $0.595^{\mathrm{b}}$ \\
\hline Height $(\mathrm{cm})$ & $162.3(9.5)$ & $163.6(8.1)$ & $0.566^{\mathrm{b}}$ \\
\hline Weight (kg) & $61.3(10.9)$ & $58.5(10.5)$ & $0.308^{b}$ \\
\hline History of illness ${ }^{\dagger}$ & $34.4 \%$ & $50.0 \%$ & $0.213^{c}$ \\
\hline Performance status $(0)^{\ddagger}$ & $96.9 \%$ & $100.0 \%$ & $1.000^{\mathrm{a}}$ \\
\hline Clinical stage (less than II) & $62.5 \%$ & $63.3 \%$ & $0.946^{\mathrm{c}}$ \\
\hline Laparoscopic Surgery & $56.3 \%$ & $50.0 \%$ & $0.622^{c}$ \\
\hline Operating time (mins) & $294.8(80.0)$ & $301.0(87.7)$ & $0.772^{b}$ \\
\hline \multicolumn{4}{|l|}{ Outcomes (3-week follow up) } \\
\hline Feeding start day & $1.1(0.3)$ & $3.7(1.3)$ & $0.000^{d}$ \\
\hline Days reaching the specified oral energy intake\# & $6.1(2.9)$ & $9.4(7.4)$ & $0.028^{\mathrm{d}}$ \\
\hline Length of stay (days) & $10.4(2.5)$ & $12.8(3.8)$ & $0.005^{\mathrm{d}}$ \\
\hline Adverse event ${ }^{+}$ & $9.4 \%$ & $23.3 \%$ & $0.176^{\mathrm{a}}$ \\
\hline
\end{tabular}

Notes. Numerical value: mean (SD), EOF: early oral feeding, COF: conventional oral feeding;

"experience of diseases prior to gastrectomy, " normal activity by ECOG performance status;

\# energy intake more than $700 \mathrm{kcal} / \mathrm{day},{ }^{+}$grade I or higher by Clavien-Dindo Classification;

${ }^{\mathrm{a}}$ Fisher's exact test, ${ }^{\mathrm{b}}$ t-test, ${ }^{\mathrm{c}}$ chi-square test, ${ }^{\mathrm{d}}$ t-test (Welch).

\subsection{Effectiveness}

Quality of life among the subjects was measured using EuroQoL-5Dimentions-3Levels (EQ-5D-3L) (EuroQol Group, 1990). A questionnaire survey of EQ-5D-3L was carried out among all the patients on each day during a 3-week follow-up period. EQ-5D-3L responses were transformed to utility values according to the utility scoring algorithms from the general population survey (Tsuchiya et al., 2002). Utility value is preference based quality of life, which evaluates the value of health state in a single score (e.g., death $=0$, perfect health $=1.0$, as anchor points).

As a health outcome measure in this economic evaluation, quality-adjusted life day (QALD) was used. QALD combines two attributes (i.e., quality of life and length of life) into a composite measure of health. QALDs in each patient were estimated through weighting each day by its utility value. Namely, a number of QALDs = a number of days of life $\mathrm{x}$ utility value. In economic evaluation, quality-adjusted life year (QALY) is most widely used (Drummond et al., 2015). However, when the change is much less than one QALY, QALY is usually expressed in days (i.e., QALD) (Ioannidis \& Graber, 2011). QALDs can be simply converted to QALYs by dividing them by 365 .

\subsection{Cost}

Costs incurred for resources used during follow-up after gastrectomy were estimated from trial data. Resource utilization was derived from individual patient data. Items for direct costs such as nutrition, hospitalization and treatment of adverse events are examined. For these direct costs, the National Health Insurance reimbursement list and drug price were used (Jiho, 2010). The cost of hospitalization per day is \$264.1 (hospitalization days 1-10), $\$ 195.2$ (days 11-20), and $\$ 166.0$ (days 21-33). There are two types of the costs of nutrition. While the commercial cost for iEAT was used from a societal perspective, insurance prices were used from a payer's perspective. The reason is that iEAT is not admitted yet for the national insurance plan. The costs per day for iEAT varied from 
$\$ 10.3$ to $\$ 12.1$ depending on their contents during POD 1 and POD 3. In contrast, the cost per day for conventional oral feeding reimbursed by the National Health Insurance was $\$ 6.3$.

On the other hand, as mentioned before, indirect costs (e.g., time costs or production loss among patients) were examined in a base case analysis. Indirect costs per patient were calculated by multiplying postoperative hospitalization days, and wages and employment rate for each day. Wages per day were estimated by the Basic Survey on Wage Structure in Japan (Ministry of Health, Labour and Welfare Japan, 2016), and employment rate was estimated by the Labor Force Survey in Japan (Ministry of Internal Affairs and Communications Japan, 2016). In addition, since there are several concerns about the inclusion of indirect costs (Drummond et al., 2015), indirect costs were excluded from the other social perspective in a sensitivity analysis.

Mean costs and their standard deviation per patient were calculated by summing up the costs listed above. All costs were converted from Japanese yen to US dollars based on Organization for Economic Co-operation and Development (OECD) purchasing power parity in $2016(\$ 1=\backslash 101.6)$ (Organization for Economic Co-operation and Development, 2016). Discounting for the time value of money was applied to neither costs nor effectiveness, since intervention and follow-up were done within a short period (Drummond et al., 2015).

\subsection{Sensitivity Analysis}

The uncertainty of the results was explored by stochastic and qualitative sensitivity analyses of important factors (Drummond et al., 2015; Briggs, 2004; Glick, Briggs, \& Polsky, 2001). Firstly, As to a base case, the impact of uncertainty on the estimated cost (e.g., hospitalization and nutrition) and effectiveness (QALDs) due to the stochastic nature of sampled data was analyzed by a stochastic sensitivity analysis, applying a bootstrap re-sampling technique (i.e., 3000 times) to both costs and effectiveness. Its results were shown as a contour curve, according to the distribution density of incremental cost-effectiveness between the EOF and COF groups (Briggs, 2004; Glick et al., 2001).

Secondly, as qualitative sensitivity analysis, three perspectives for economic evaluation were examined (i.e., a social perspective including indirect costs (a base case), a social perspective excluding indirect costs, and a payer's perspective). A base case analysis in this paper adopted a social perspective including indirect costs. Even though indirect costs should theoretically be included from a social perspective (Drummond et al., 2015), there has been debate about treating and estimating indirect costs (Drummond et al., 2015; Jönsson, 2009; Jacob \& Fassbender, 1998). So, secondly, costs were estimated from a social perspective excluding indirect costs (i.e., a conservative point of view). Finally, from a payer's perspective, economic evaluation was conducted.

Therefore, as to costs, a social perspective (Drummond et al., 2015) including indirect costs included both direct and indirect costs. It should be noticed that it used commercial prices for iEAT as nutritional costs among the EOF group. On the other hand, a social perspective excluding indirect costs excluded indirect costs from the costs by a social perspective including indirect costs. The third payer's perspective (Drummond et al., 2015) included only direct costs. In this case, only reimbursement rates for nutritional care were used among both the EOF and COF groups.

The commercial prices for iEAT are higher than those reimbursement rates. From a payer's perspective, differences between these costs were not considered, and transferred to patients or in some cases healthcare providers.

\subsection{Statistical Analysis}

As to statistical analysis, quantitative data comparison between two groups was analyzed by using t-test, or t-test (Welch) when the variance of both groups is not the same. This analysis was based on the normal distribution according to the central limit theorem, since the sample sizes of both groups exceeded 30 . However, simultaneously, data was analyzed by using Mann-Whitney $U$ test, without any assumptions about the distribution of both groups. If there was a large discrepancy between these tests, additional information based on Mann-Whitney $U$ test was given. In addition, $95 \%$ confidence intervals for incremental effectiveness and costs were shown. On the other hand, categorical data was analyzed by using chi-square test, or Fisher's exact test when the sample size of each cell in a table was less than 10. All statistical analyses were performed using SPSS 16.0J and TreeAge software.

\section{Results}

\subsection{Effectiveness}

The mean QALDs in each group are shown in Table 2. The mean QALDs for the EOF and the COF groups were 14.93 and 14.19 , respectively. Incremental effectiveness of the EOF group compared with the COF group was 0.74 
QALDs ( $\mathrm{p}=0.359)$. EOF increased 0.74 QALDs (i.e., $2.03 \times 10^{-3}$ QALYs) per patient, compared with COF. This difference was not statistically significant.

Table 2. Incremental effectiveness and costs of EOF compared with COF per person

\begin{tabular}{|c|c|c|c|c|}
\hline Item & $\operatorname{EOF}(\mathbf{N}=32)$ & $\operatorname{COF}(\mathrm{N}=30)$ & $\begin{array}{l}\text { Incremental effectiveness } \\
\text { and costs } \\
\text { (95\% confidence intervals) }\end{array}$ & p value \\
\hline \multicolumn{5}{|l|}{ Effectiveness } \\
\hline QALDs & $14.93(3.26)$ & $14.19(3.03)$ & $0.74(-0.86 \sim 2.34)$ & $0.359^{\mathrm{a}}$ \\
\hline \multicolumn{5}{|l|}{ Total costs (\$) } \\
\hline $\begin{array}{l}\text { Societal perspective including } \\
\text { indirect costs }\end{array}$ & $3177.7(808.4)$ & 3755.4 (1 214.6) & $-577.7(-1107.6 \sim-47.8)$ & $0.033^{\mathrm{b}}$ \\
\hline $\begin{array}{l}\text { Societal perspective excluding } \\
\text { indirect costs }\end{array}$ & $2707.7(538.6)$ & $3151.8(862.0)$ & $-444.1(-813.9 \sim-74.3)$ & $0.020^{\mathrm{b}}$ \\
\hline Payer's perspective & $2669.8(538.0)$ & $3151.8(862.0)$ & $-482.0(-851.7 \sim-112.3)$ & $0.012^{\mathrm{b}}$ \\
\hline
\end{tabular}

Notes. Numerical value: mean (SD), EOF: early oral feeding, COF: conventional oral feeding, QALD: quality-adjusted life day, ${ }^{\mathrm{a}}$ t-test, ${ }^{\mathrm{b}}$ t-test (Welch).

\subsection{Cost}

The mean total costs per patient in each group are shown in Table 2. From a societal perspective including indirect costs, as a base case analysis, the mean total cost per patient in the EOF and the COF groups was $\$ 3,177.7$ and $\$ 3,755.4$, respectively $(\mathrm{p}<0.05)$. Incremental cost of the EOF group compared with the COF group was -577.7 $(\mathrm{p}=0.03)$. EOF saved $\$ 577.7$ per patient, compared with COF.

The main cost component was costs of hospitalization (Table 3). That in the EOF group was statistically lower than that in the COF group $(\mathrm{p}<0.01)$. In contrast, the cost of nutrition (considering the price of iEAT) in the EOF group was higher than that in the COF group (not statistically significant). EOF increased $\$ 29.8$ per patient, compared with COF. The cost for treatment of adverse events was not observed, since they were not so severe as to require specific treatments. As to indirect costs, that in the EOF group was lower than that in the COF group, but no statistical difference was observed.

In considering both incremental effectiveness and costs, EOF was cost-saving, with non-significant increase in effectiveness (i.e., QALDs). Therefore, from a societal perspective considering indirect costs, EOF was dominant, or at least weakly dominant (Drummond et al., 2015), compared with COF.

Table 3. Cost components of EOF and COF per person

\begin{tabular}{|c|c|c|c|}
\hline Item & $\operatorname{EOF}(\mathrm{N}=32)$ & $\operatorname{COF}(\mathrm{N}=30)$ & p value \\
\hline \multicolumn{4}{|l|}{ Direct costs $(\$)$} \\
\hline Hospitalization & 2475.0 & 2948.9 & $0.007^{\mathrm{b}}$ \\
\hline Nutrition & $232.7(55.6)$ & $202.9(78.9)$ & $0.089^{\mathrm{a}}$ \\
\hline Adverse event treatment & NA & NA & \\
\hline \multicolumn{4}{|l|}{ Indirect costs $(\$)$} \\
\hline Return to work & $470.0(401.0)$ & $603.6(536.6)$ & $0.269^{\mathrm{a}}$ \\
\hline
\end{tabular}

Notes. Numerical value: mean (SD), EOF: early oral feeding, COF: conventional oral feeding, a t-test, b t-test (Welch).

\subsection{Sensitivity Analysis}

The results of a stochastic sensitivity analysis are shown in Figure 1, which shows scatter plots of incremental cost and effectiveness between the EOF and COF groups. In this Figure, higher density of distribution for scatter plots 
of cost and effectiveness, based on 3,000 samples, was shown as a darker area in a contour curve. More than $31 \%$ of the points resided in the southeast quadrant (i.e., cost saving and greater effectiveness). Also, if willingness to pay for additional QALY was set at $\$ 5 \times 10^{4}$ (Grosse, 2008), more than $58 \%$ of the points resided below this threshold, which means that EOF is cost-effective or efficient.

The results of qualitative sensitivity analyses are shown in Table 2. As to the societal perspective excluding indirect costs, the mean total costs in the EOF group were significantly lower than those in the COF group $(\mathrm{p}<0.05)$. This is also true from a payer's perspective $(\mathrm{p}<0.05)$.

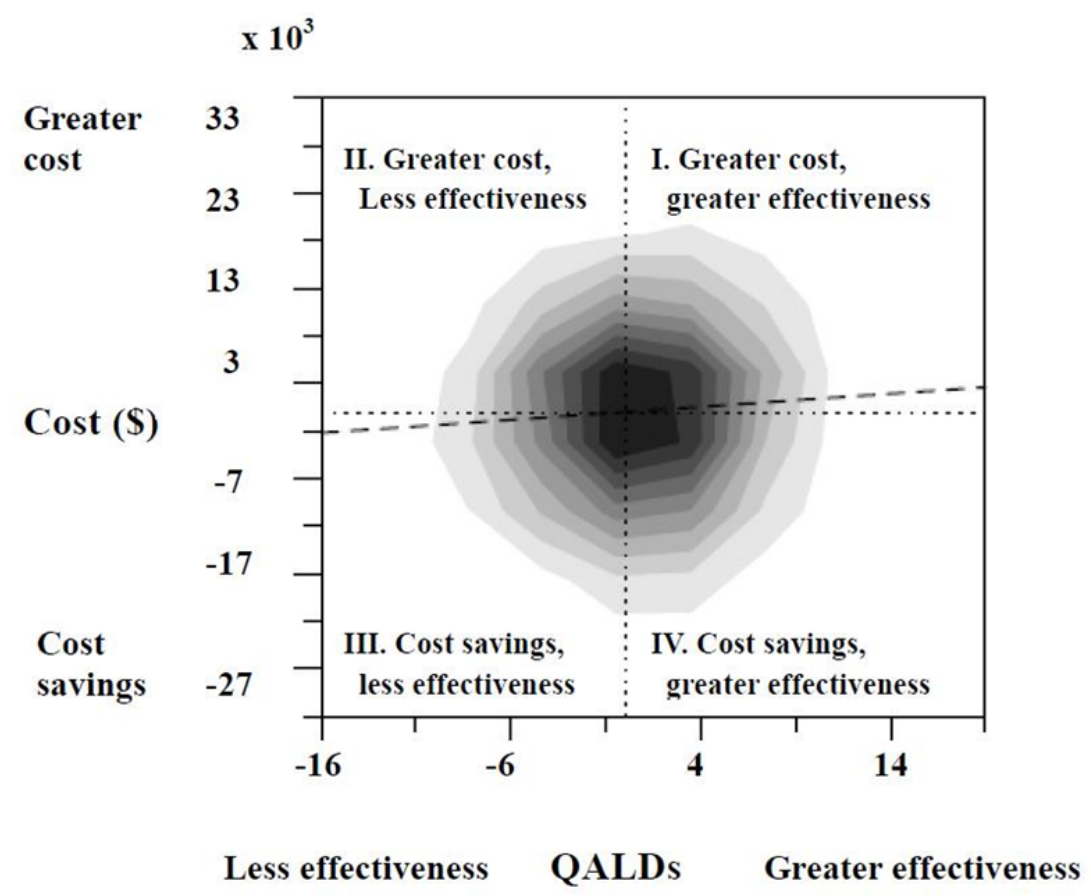

Figure 1. Stochastic sensitivity analysis

Incremental cost-effectiveness contour curve shows the relative concentration of points in the scatter plot for cost-effectiveness ratios, with a range of shades. An oblique line shows that cost-effectiveness ratio is $\$ 5 \times 10^{4}$ per QALY.

\section{Discussion}

Evidence from systematic reviews (Liu et al., 2014; Tweed et al., 2019; Willcutts et al., 2016) indicates that EOF following gastrectomy for gastric cancer is effective for shortening the length of hospital stay without increasing averse events. However, the cost-effectiveness of EOF following gastrectomy has not been well explored. The only exception is a systematic review for ERAS, which indicated a reduction in hospital costs ( $\mathrm{Li}$ et al., 2017). However, it has not fully examined both effectiveness and costs comprehensively, but only roughly mentioned costs.

Our study is the first attempt to evaluate the cost-effectiveness of EOF following total gastrectomy based on a RCT. The study adopted the societal perspective and showed that EOF after gastrectomy saved costs with small improvement of health outcomes (i.e., QALDs), compared with COF (Table 2). That is to say, EOF dominated COF. This dominance is not so strong, rather weak (Drummond et al., 2015), since increase in effectiveness is not statistically significant. According to qualitative sensitivity analysis on perspectives of economic evaluation, from both societal perspective, with or without including indirect costs, and payer's perspective, this result was confirmed. The most important cost component was the cost of hospitalization, of which the absolute value was around 10 times or 6 times higher than the costs of nutrition and indirect costs, respectively. This cost is clearly dependent on the length of hospital stay.

To estimate the uncertainty of this result due to sampling bias, stochastic sensitivity analyses (Drummond et al., 2015; Briggs, 2004; Glick et al., 2001) were performed (Figure 1). The cost-effectiveness contour curve showed 
that more than $31 \%$ of cost-effectiveness plots located in the southeast quadrant (cost savings and greater effectiveness) on the cost-effectiveness plane. This result shows that the dominant cost-effectiveness of EOF is relatively robust.

This cost saving result could be ranked near the top of a league table of cost-effectiveness in health care, since less than $20 \%$ of published cost-effectiveness studies showed cost savings (Russell, 2009). Therefore EOF after total gastrectomy among gastric cancer patients is an efficient approach to postoperative management and can be considered as a cost-effective intervention to be accepted for wide use in Japan. Recently, in Japan, the application of cost-effectiveness of health technologies into health policy making has been tried. However, this is only applied for adjustment of the premium price based on the existing reference pricing system. Also, the subject drugs are restricted to new drugs with high price and high volume.

It is pointed out that this policy-making process is not evidence-based and is biased in many points (Hisashige, 2018). In addition, this analysis is primarily based on a payer's perspective for administrative approval. It would not fully capture the value of health technologies from a societal perspective. Moreover, although the administrative guidelines in Japan have been released (Study Team for "Establishing Evaluation Methods, Data Standardization, and Assessment Systems Toward the Application of Economic Evaluation of Healthcare Technologies to Governmental Policies", 2016), they mostly follow existing guidelines and issues unsolved among them have not been examined and still remain.

There are several points for discussions in the analysis that should be commented on, and the results should be treated with caution. First, the analysis was based on a small RCT (Shimizu et al., 2018). It would be vulnerable to uncertainty (Hackshaw, 2008). Systematic reviews of EOF after gastrectomy indicated that large RCTs with high quality are required to confirm current clinical evidence (Liu et al., 2014; Willcutts et al., 2016). It is pointed out that the main problem in each small study is that it produces a broad confidence interval (or imprecise estimate) of treatment effect, compared with a large study (Hackshaw, 2008). A funnel plot of studies shows that estimated effects among small studies scattered widely (i.e., a wide range of positive or negative results), even if there is no bias (Sedgwick \& Marston, 2015). On the other hand, large studies often produce results far from definitive (Guyatt, Mills, \& Elboume, 2008). Also, a recent study shows that a meta-analysis containing many small studies is better than a single large trial, even when there is some publication bias (Borm \& Donders, 2009). Therefore, in the current paradigm, multiple small studies are conducted and subsequently combined in a meta-analysis with an examination of heterogeneity (IntHout, Ioannidis, Borm, \& Goeman, 2015).

As to EOF, there is nothing wrong with conducting well-designed small studies a), accumulating the results of individual trials, regardless of small or large size, is best viewed as providing important information that contributes to the larger body of evidence (Guyatt, Mills, \& Elboume, 2008).

Second, the time horizon for analysis is an important issue in sufficiently capturing relevant costs and health outcomes of EOF. In this study, the observation period of the trial (i.e., 3 weeks) was relatively limited, due to continuous diary survey for quality of life. As mentioned before, the length of hospital stay is a main cost factor. Since a small proportion of patients were still staying in a hospital, the time horizon in this study would underestimate the value of EOF in both hospital costs and indirect costs.

Third, the perspective of this analysis is that of a society. A societal perspective examines a broader range of costs compared with that of health care payers. A qualitative sensitivity analysis showed cost saving for EOF in both societal and payer's perspective. However, cost savings were much higher in the societal perspective including indirect costs. Also, there are some conflicts between theses perspectives. In Japan, fixed and bundling payments have been introduced to health care management in the national health insurance. Items such as nutrition support by iEAT are not listed in the national health insurance, so they are not reimbursed. Then, these costs are transferred to patients or healthcare providers. Therefore, changes in cost items due to a perspective of economic evaluation should be carefully examined.

Fourth, QALDs as a final outcome were used as economic outcome measures for the analysis. There were very few studies on quality of life for EOF or COF after gastrectomy. Hur et al. (Hur et al., 2011) used a disease specific instrument (i.e., EORTC) in evaluating oral feeding after gastric cancer surgery. However, it is not applicable in economic evaluation in healthcare. In our study, a general profile instrument (i.e., EQ-5D-3L) was used, and easily converted to utility (i.e., preference-based quality of life which evaluates the value of health states in a single score). Then, QALDs (or QALYs) can be used as a final outcome for economic evaluation.

Fifth, this study examined only EOF among total gastrectomy. A systematic review (Liu et al., 2014) showed that similar findings were found in both total gastrectomy and subtotal gastrectomy with regard to duration of hospital 
stay and other items. However, in a recent RCT in Japan (Shimizu et al., 2018), EOF did not shorten the length of hospital stay in distal gastrectomy. So, it might still be needed to carefully assess cost-effectiveness of EOF for both total and partial gastrectomy, independently.

Sixth, this study showed the potential benefits of iEAT diet for EOF. In the past, as EOF, water, fluids or liquid diets have been widely used (Liu et al. 2014). Comparative effectiveness between iEAT and other diets for EOF has not been evaluated yet. Therefore, research exploring appropriate and optimal diet for EOF would be needed.

Also, it should be noticed that several components of ERAS have been introduced, more or less, into clinical settings in Japan (Kaibori, Miyata, Yoshii, \& Fukushima, 2020). In our study, since components of ERAS except for EOF were randomized, they would not affect its results. However, the space for improvement by EOF alone seems to be limited.

Lastly, most of the studies on EOF following gastrectomy have been carried out in East Asian countries, such as Japan, Korea and China (Liu et al., 2014; Willcutts et al., 2016). It is clear that there is a paucity of studies in the Western countries. This seems to be due to the difference of incidence rates of gastric cancer. Also, health outcomes of EOF following gastrectomy would be influenced by racial, environmental, life-style or medical differences between the Western and Eastern countries. However, one RCT on EOF for total gastrectomy, conducted in the US showed a decreased length of stay (Selby, Rifkin, Yoon, Ariyan, \& Strong, 2016). Therefore, the results of our study would be a helpful reference to the US or other Western countries. Since in general, economic evaluation can not be readily transferable to other countries (Drummond et al., 2009), economic evaluation of EOF should be carried out in each country.

\section{Conclusion}

In considering both incremental effectiveness and costs, economic evaluation of EOF following total gastrectomy showed that EOF was cost-saving, with non-significant increase in effectiveness (i.e., QALD). This cost saving result could be ranked quite highly in a league table of cost-effectiveness in health care. This result can contribute to strengthen the evidence for wide use of EOF.

\section{Declarations}

\section{Ethics Approval and Consent to Participate}

The study was based on a clinical trial approved by the institutional review board at each of the study sites and was performed in accordance with the principles of the Declaration of Helsinki. Informed consent was obtained from all the patients and/or guardians.

\section{Funding}

No funding was received for this study.

\section{Acknowledgements}

Not applicable.

\section{Role of Each Author in the Work}

AH: study concept and design, acquisition, analysis and interpretation of economic data, and preparation of manuscript

NS and YS: acquisition and analysis of subject and clinical data

\section{Competing Interests Statement}

The authors declare that there are no competing or potential conflicts of interest.

\section{References}

Beamish, A. J., Chan, D. S., Blake, P. A., Karran, A., \& Lewis, W. G. (2015). Systematic review and meta-analysis of enhanced recovery programmes in gastric cancer surgery. Int $J$ Surg, 19, 46-54. https://doi.org/10.1016/j.jisu.2015.05.021

Borm, G. F., \& Donders, R. (2009). A treatment should be evaluated by small trials. J Clin Epidemiol, 62, 887-889.

Bray, F., Ferlay, J., Soerjomataram, I., Siegel, R. L., Torre, L. A., \& Jemal, A. (2018). Global cancer statistics 2018: GLOBOCAN estimates of incidence and mortality worldwide for 36 cancers in 185 countries. CA Cancer $J$ Clin, 68, 394-424. https://doi.org/10.3322/caac.21492

Briggs, A. H. (2004). Statistical approaches to handling uncertainty in health economic evaluation. Eur $J$ Gastroenterol Hepatol, 16, 551-561. https://doi.org/10.1097/00042737-200406000-00007 
Coburn, N., Cosby, R., Klein, L., Knight, G., Malthaner, R., Mamazza, J., ... Ringash, J. (2018). Staging and surgical approaches in gastric cancer: A systematic review. Cancer Treat Rev, 63, 104-115. https://doi.org/10.1016/j.ctrv.2017.12.006

Drummond, M. F. (2015). Methods for the economic evaluation of health care programmes (4th ed.). Oxford Univ Press.

Drummond, M., Barbieri, M., Cook, J., Glick, H. A., Lis, J., Malik, F., ... Severens, J. (2009). Transferability of economic evaluations across jurisdictions: ISPOR Good Research Practices Task Force report. Value Health, 12, 409-418. https://doi.org/10.1111/j.1524-4733.2008.00489.x

EuroQol Group. (1990). EuroQol--a new facility for the measurement of health-related quality of life. Helath Policy, 16, 199-208. https://doi.org/10.1016/0168-8510(90)90421-9

Fukatsu, K. (2019). Role of nutrition in gastroenterological surgery. Ann Gastroenterol Surg, 3, $160-168$. https://doi.org/10.1002/ags3.12237

Glick, H. A., Briggs, A. H., \& Polsky, D. (2001). Quantifying stochastic uncertainty and presenting results of cost-effectiveness analyses. Expert Rev Pharmacoecon Outcomes Res, 1, 25-36. https://doi.org/10.1586/14737167.1.1.25

Gramlich, L. M., Sheppard, C. E., Wasylak, T., Gilmour, L. E., Liunggvist, O., Basualdo-Hammond, C., \& Nelson, G. (2017). Implementation of enhanced recovery after surgery: a strategy to transform surgical care across a health system. Implement Sci, 12, 67-84. https://doi.org/10.1186/s13012-017-0597-5

Grosse, S. D. (2008). Assessing cost-effectiveness in healthcare: history of the $\$ 50,000$ per QALY threshold. Expert Rev Pharmacoecon Outcomes Res, 8,165-178. https://doi.org/10.1586/14737167.8.2.165

Guyatt, G., Mills, E. J., \& Elboume, D. (2008). In the era of systematic reviews, does the size of an individual trial still matter. PLos Med., 5, 1-e4. https://doi.org/10.1371/journal.pmed.0050004

Hackshaw, A. (2008). Small studies: strengths and limitations. Eur Respr J, 32, 1141-1143. https://doi.org/10.1183/09031936.0013648.

Higashiguchi, T. (2013). Novel diet for patients with impaired mastication evaluated by consumption rate, nutrition intake, and questionnaire. Nutrition, 29, 858-864. https://doi.org/10.1016/j.nut.2012.12.016

Hisashige, A. (2018). The trends in health policy for cost-effectiveness of health technologies in Japan, part 1. Jpn $J$ Health Economic Policy, 30, 20-31. (in Japanese)

Hur, H., Kim, S. G., Shim, J. H., Song, K. Y., Kim, W., Park, C. H., \& Jeon, H. M. (2011). Effect of early oral feeding after gastric cancer surgery: a result of randomized clinical trial. Surgery, 149, 561-568. https://doi.org/10.1016/j.surg.2010.10.003

IntHout, J., Ioannidis, J. P. A., Borm, G. F., \& Goeman, J. J. (2015). Small studies are more heterogeneous than large ones: a meta-meta-analysis. $J$ Clin Epidemiol, $68, \quad$ 860-869. https://doi.org/10.1016/j.jclinepi.2015.03.017

Ioannidis, J. P., \& Garber, A. M. (2011). Individualized cost-effectiveness analysis. PLoS Med, 8, e1001058. https://doi.org/10.1371/journal.pmed.1001058.

Jacobs, P., \& Fassbender, K. (1998). The measurement of indirect costs in the health economics evaluation literature. A review. Int J Technol Assess Health Care, 14, 799-808. https://doi.org/10.1017/s0266462300012095

Jiho. (2010). Encyclopedia of drugs listed for insurance. Tokyo: Jiho Inc (in Japanese).

Jönsson, B. (2009). Ten arguments for a societal perspective in the economic evaluation of medical innovations. Eur J Health Econ, 10, 357-359. https://doi.org/10.1007/s10198-009-0173-2

Kaibori, M., Miyata, G., Yoshii, K., \& Fukushima, R. (2020). Preoperative management for gastrointestinal surgery after instituting interventions initiated by the Japanese Society of Surgical Metabolism and Nutrition. Asian J Surg, 43(1), 124-129. https://doi.org/10.1016/j.asjsur.2019.02.007

Li, Z., Wang, Q., Li, B., Bai, B., \& Zhao, Q. (2017). Influence of enhanced recovery after surgery programs on laparoscopy-assisted gastrectomy for gastric cancer: a systematic review and meta-analysis of randomized control trials. World J Surg Oncol, 15:207-216. https://doi.org/10.1186/s12957-017-1271-8.

Liu, X., Wang, D., Zheng, L., Mou, T., Liu, H., \& Li, G. (2014). Is early oral feeding after gastric cancer surgery 
feasible? A systematic review and meta-analysis of randomized controlled trials. PLoS One, 9, e112062. https://doi.org/10.1371/journal.pone.0112062

Ministry of Health, Labour and Welfare Japan. (2016). Basic survey on wage structure. Retrieved from https://www.mhlw.go.jp/english/database/db-l/basicsurvey/xls/2016.xls

Mortensen, K., Nilsson, M., Slim, K., Schäfer, M., Mariette, C., Braga, M., ..., \& Lassen, K. (2014). Consensus guidelines for enhanced recovery after gastrectomy: Enhanced Recovery After Surgery (ERAS ${ }^{\circledR}$ ) Society recommendations. Br J Surg, 101, 1209-1229. https://doi.org/10.1002/bjs.9582

OECD. GDP PPPs and derived indices for all OECD countries. (2016). OECD main economic indicators, OECD. Retrived from http://www.oecd.org/sdd/purchasingpowerparitiespppsdata.htm

Paton, F., Chambers, D., Wilson, P., Eastwood, A., Craig, D., Fox, D., ..., \& McGinnes, E. (2014). Effectiveness and implementation of enhanced recovery after surgery programmes: a rapid evidence synthesis. BMJ Open, 4, e005015. https://doi.org/ 10.1136/bmjopen-2014-005015

Rawla, P., \& Barsouk, A. (2019). Epidemiology of gastric cancer: grobal trends, risk factors and prevention. Prz Gastroenterol, 14, 26-38. https://doi.org/10.5114/pg.2018.80001

Russell, L. B. (2009). Preventing chronic diseases: an important investment, but don't count on cost savings. Health Aff (Millwood), 28, 42-45. https://doi.org/10.1377/hlthaff.28.1.42.

Sedgwick, P., \& Marston, L. (2015). How to read a funnel plot in a meta-analysis. BMJ. 16, 351, h4718. https://doi.org/10.1136/bmj.h4718

Selby, L. V., Rifkin, M. B., Yoon, S. S., Ariyan, C. E., \& Strong, V. E. (2016). Decreased length of stay and earlier oral feeding associated with standardized postoperative clinical care for total gastrectomies at a cancer center. Surgery, 160, 607-612. https://doi.org/10.1016/j.surg.2016.04.036

Shimizu, N., Oki, E., Tanizawa, Y., Suzuki, Y., Aikou, S., Kunisaki, T., ..., \& Seto, Y. (2018). Effect of early oral feeding on length of hospital stay following gastrectomy for gastric cancer: a Japanese multicenter, randomized controlled trial. Surg Today, 48, 865-874. https://doi.org/10.1007/s00595-018-1665-4

Statistics Bureau, Ministry of Internal Affairs and Communications Japan. (2016). Labor force survey. Retrieved from http://www.stat.go.jp/english/data/roudou/report/2016/index.html

Study Team for establishing evaluation methods, data standardization, and assessment systems toward the application of economic evaluation of healthcare technologies to governmental policies. (2016). Guideline for preparing cost-effectiveness evaluation to the central social insurance medical council. Retrived from https://ars.els-cdn.com/content/image/1-s2.0-S1098301516313067-mmc1.pdf

The Editorial Board of the Cancer Statistics in Japan, eds. Cancer Statistics in Japan (2018). Tokyo: Foundation for Promotion of Cancer Research, 2019 (in Japanese).

Tsuchiya, A., Ikeda, S., Ikegami, N., Nishimura, S., Sakai, I., Hamashima, C., ..., \& Tamura, M. (2002). Estimating an EQ-5D population value set: the case of Japan. Health Econ, 11, 341-353. https://doi.org/10.1002/hec.673

Tweed, T., van Eijiden, Y., Tegels, J., Brenkman, H., Ruurda, J., van Hillegersberg, R., ... , \& Soot, J. (2019). Safety and efficacy of early oral feeding for enhanced recovery following gastrectomy for gastric cancer: A systematic review. Surg Oncol, 28, 88-95. https://doi.org/10.1016/j.surinc.2018.11.017

Willcutts, K. F., Chung, M. C., Erenberg, C. L., Finn, K. L., Schirmer, B. D., \& Byham-Gray, L. D. (2016). Early oral feeding as compared with traditional timing of oral feeding after upper gastrointestinal surgery: a systematic review and meta-analysis. Ann Surgery, 264, 54-63. https://doi.org/10.1097/SLA.0000000000001644.

\section{Copyrights}

Copyright for this article is retained by the author(s), with first publication rights granted to the journal.

This is an open-access article distributed under the terms and conditions of the Creative Commons Attribution license (http://creativecommons.org/licenses/by/4.0/). 